\title{
QUÍMICA Y DISTRIBUCIÓN DE UMBILICARIA FREYI, U. GRISEA Y U. HIRSUTA EN LA PENÍNSUla IBÉRICA
}

\author{
Leopoldo G. SANCHO, Estela SERIÑÁ, \\ Rosario ARROYO \& Esteban MANRIQUE
}

\begin{abstract}
RESUMEN: Se realiza un estudio químico comparado de Umbilicaria freyi Codogno \& al., una nueva especie descrita recientemente, y de sus especies afines $U$. hirsuta (Sw.) Ach. y $U$. grisea Hoffm. Se aportan nuevos datos sobre la distribución y ecología de estos tres táxones en la Península Ibérica.

Palabras clave: Liquenes, Umbilicaria, química, distribución.

ABSTRACT: Chemistry and distribution of Umbilicaria freyi, U. grisea and U. hirsuta on the Iberian península: This paper reports a comparative chemical study of Umbilicaria freyi Codogno \& al., a species recently described, and the related species $U$. hirsuta $(\mathrm{Sw}$.) Ach. and $U$. grisea Hoffm. New data about the distribution and ecology of these three taxa on the Iberian peninsula are presented.
\end{abstract}

Key words: Lichens, Umbilicaria, chemistry, distribution.

\section{INTRODUCCIÓN}

Aunque el género Umbilicaria ha sido estudiado en Europa por numerosos autores aún subsisten problemas taxonómicos, especialmente entre las especies con un óptimo de distribución mediterráneo. Recientemente ha sido descrita, a partir de poblaciones italianas, un nueva especie, Umbilicaria freyi Codogno, Poelt \& Puntillo (1989), estrechamente relacionada con U. hirsuta (Sw.) Ach. y U. grisea Hoffm.

El principal carácter diferencial de Umbilicaria freyi consiste en el desarrollo de esquizidios en la parte superior del talo como forma de reproducción asexual (Fig. 1A, B). En el género Umbilicaria los esquizidios sólo habían sido descritos hasta ahora en $U$. thamnodes, especie endémica del Himalaya (Poelt, 1977). U. hirsuta y $U$. grisea producen también a partir del córtex superior propágulos vegetativos, denominados por Codogno \& al. (1989) parasoredios (Fig. 1C), diferentes, desde un punto de vista ontogénico y morfológico, de los auténticos soredios. 

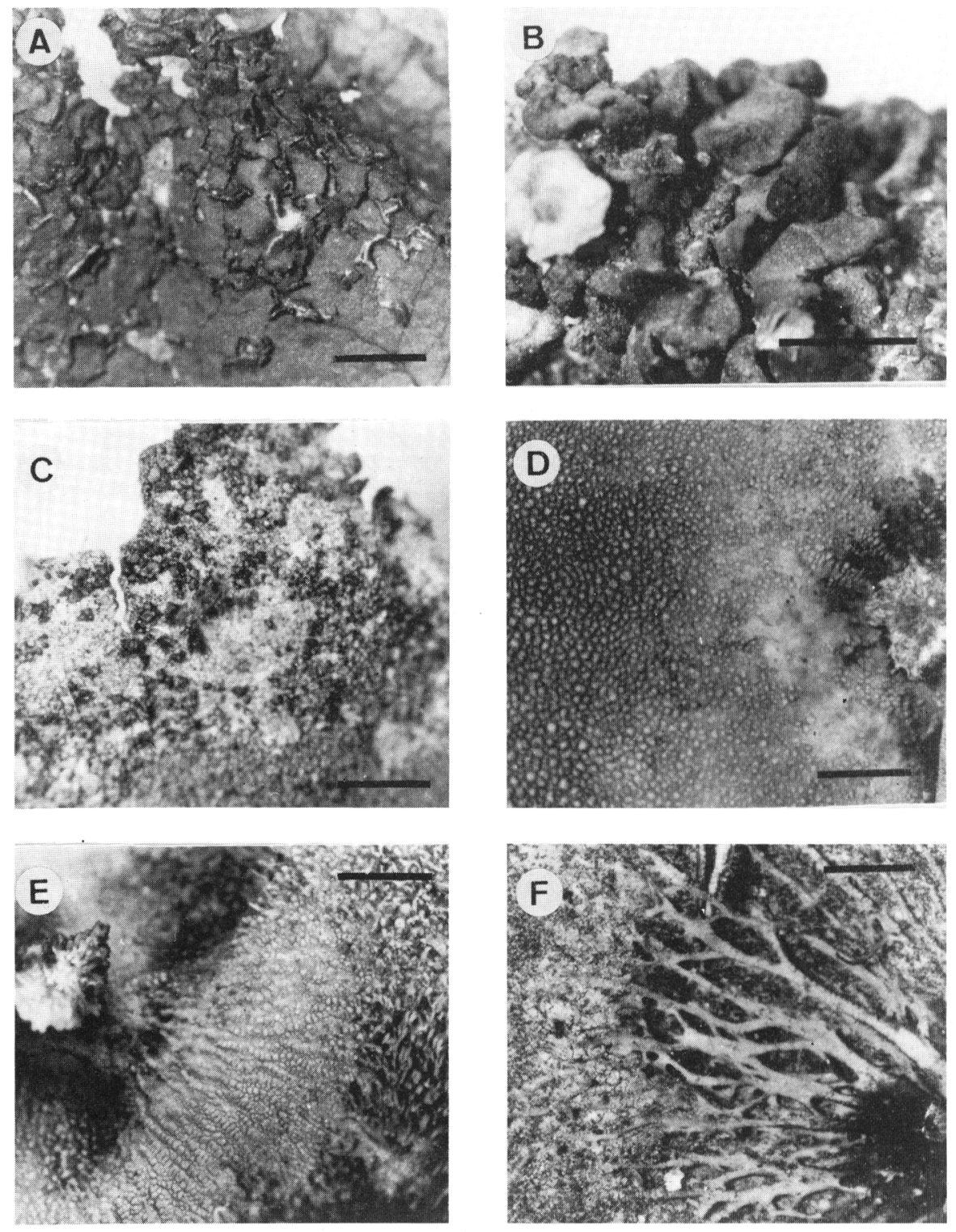

Fig. 1.- Aspecto del talo de Umbilicaria. A, B y E, U.freyi: A, B cara superior con esquizidios; $\mathrm{E}$, cara inferior. C y D, U. grisea: $\mathrm{C}$, cara superior con parasoredios; $\mathrm{D}$, cara inferior. F, U. hirsuta: cara inferior. Escala $=1 \mathrm{~mm}$. 
La distribución de Umbilicaria hirsuta y U. grisea abarca toda Europa, desde la Península Ibérica hasta Escandinavia, si bien $U$. grisea no alcanza latitudes tan septentrionales como $U$. hirsuta y se comporta además como su vicariante altitudinal en los pisos basales (Wirth, 1980). El área de distribución de $U$. freyi queda prácticamente restringida al Mediterráneo occidental, aunque se conocen algunas localidades algo más septentrionales (Codogno \& al., 1989; Codogno \& Sancho, 1990). Tanto $U$. freyi como $U$. grisea están ausentes o son muy puntuales en las zonas montañosas de orogenia alpina (Codogno \& Sancho, 1990).

En este trabajo nos planteamos aportar nuevos datos sobre la distribución de este grupo en la Península Ibérica, así como intentar aclarar sus posibles afinidades o diferencias mediante el análisis químico de distintas poblaciones repartidas en un área geográfica extensa.

\section{MATERIAL Y MÉTODOS}

Se han analizado 30 ejemplares procedentes de 24 localidades mediante técnicas de cromatografía líquida de alta resolución (HPLC). Se ha utilizado un cromatógrafo Perkin Elmer Series 10, equipado con una columna analítica Spherisorb 55 ODS $25 \mathrm{Im}$ de $25 \mathrm{~cm}$ x $4.6 \mathrm{~mm}$; la fase móvil es una mezcla de metanol-agua-ácido acético (75:25:2). La detección se efectuó con un espectrofotómetro Lambda 1 fijado a $270 \mathrm{~nm}$. Los cálculos se realizaron con un registrador-integrador LCI 100 PerkinElmer. El flujo del disolvente fue de $1 \mathrm{ml} / \mathrm{min}$ (1200 psi) y el volumen de inyección constante $(6 \mu \mathrm{m})$. La extracción de las sustancias liquénicas se realizó con acetona a temperatura ambiente durante 10 minutos.

Para el análisis de componentes principales se han seleccionado 24 talos, 8 de cada una de las especies.

Los ejemplares analizados de las distintas especies proceden de las siguientes localidades:

Umbilicaria hirsuta: España: Madrid: Sierra de Guadarrama, Siete Picos, 2120 m, 14 de Septiembre de 1988, L.G. Sancho \& F. Valladares (MAF). Avila: Sierra de Gredos, 1870 m, 12 de Junio de 1989, L.G. Sancho \& F. Valladares (MAF); Sierra de la Paramera, Pico Zapatero, 2100 m, 30 de Abril de 1989, L.G. Sancho (MAF). Italia: Calabria: Reggio Calabria, Aspromonte, Pietra Impicata, 1750 m, 12 de Julio de 1988, J. Poelt, M. Codogno, D. Puntillo \& L.G. Sancho (MAF); Cosenza, 1200 m, 11 de Julio de 1988, J. Poelt, M. Codogno, D. Puntillo \& L.G. Sancho (MAF).

Umbilicaria grisea: España: Madrid: Torrelodones, 870 m, 21 de Septiembre de 1988, L.G. Sancho (MAF); Sierra de Guadarrama, El Escorial, 1050 m, 9 de Enero de 1988, C. Sierra Rico (MAF); La Peñota, 1400 m, 4 de Febrero de 1978, L.G. Sancho (MAF). Toledo: San Pablo de los Montes, 1 de Octubre de 1988, L. Balaguer (MAF). Salamanca: Las Batuecas, 2 de Enero de 1987, L.G. Sancho (MAF). Suiza: Otzaler Alpen, 1800 m, 1968, G. Feige (MAF).

Umbilicaria freyi: España: Madrid: Sierra de Guadarrama, Pto. de la Morcuera, 1500 m, 16 de Noviembre de 1988, L.G. Sancho (MAF); Sierra de la Cabrera, 1350 m, 21 de Septiembre de 1988, L.G. Sancho (MAF). Guadalajara: Valverde de los Arroyos, Pico Ocejón, 
1400 m, 23 de Octubre de 1988, L.G. Sancho (MAF). Palencia: Cardaño de Arriba, 1550 m, 10 de Septiembre de 1985, A. Terrón. Italia: Calabria: Reggio Calabria, Aspromonte, Puntone Gramolara, 1440 m, 12 de Julio de 1988, J. Poelt, M. Codogno, D. Puntillo \& L.G. Sancho (MAF).

\section{DISTRIBUCIÓN Y ECOLOGÍA}

Las tres especies estudiadas pueden considerarse como frecuentes y abundantes en la Península Ibérica, siendo la altitud el principal factor discriminante. Umbilicaria grisea único representante del género Umbilicaria de comportamiento claramente termofítico, ocupa en la Península Ibérica, con preferencia, los pisos bioclimáticos meso- y supramediterráneo inferior, aunque puede encontrarse puntualmente hasta en el oromediterráneo inferior (en la terminología y concepto de los pisos bioclimáticos seguimos a Rivas-Martínez, 1988), su área de distribución en la Península Ibérica es por tanto la más extensa. Abarca todo el occidente peninsular, extendiéndose a lo largo del Sistema Central hasta el Moncayo, con algunas localidades extremadamente orientales en ciertas rocas ácidas del Sistema Ibérico (Albacete, Sierra del Relumbrar) y en el Montseny (Hladún, 1982). Hacia el sur alcanza las zonas basales de Sierra Nevada y la Sierra de los Filabres (Egea \& Llimona, 1981) (Fig. 2).

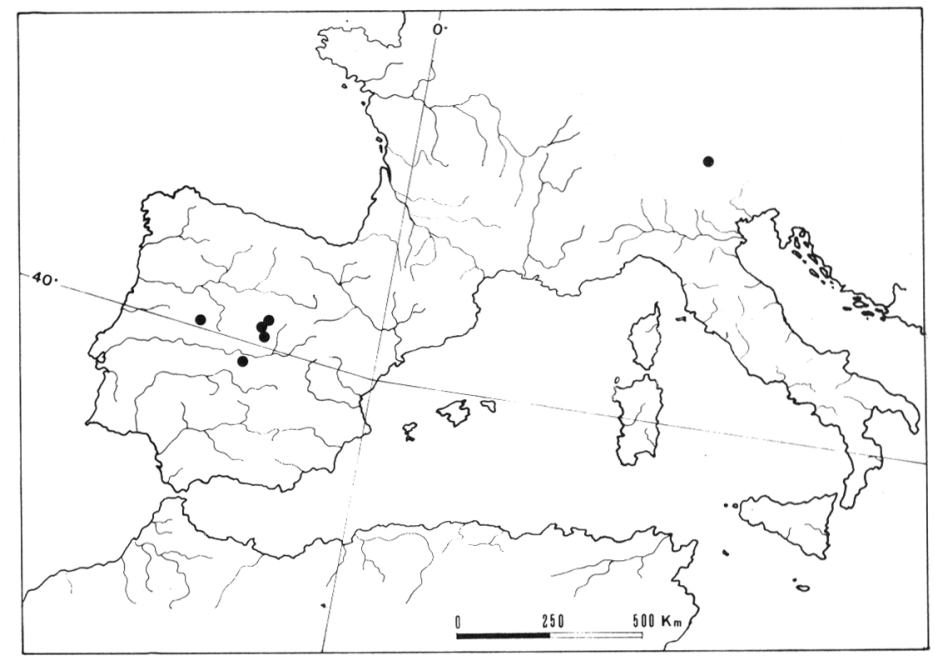

Fig. 2.- Mapa de distribución de los ejemplares analizados de Umbilicaria grisea.

Umbilicaria hirsuta es, por el contrario, una especie de preferencias alpinas. En nuestro territorio se encuentra sobre todo a partir del piso supramediterráneo superior o del montano en el caso de los Pirineos y Cordillera Cantábrica (Fig. 3). 


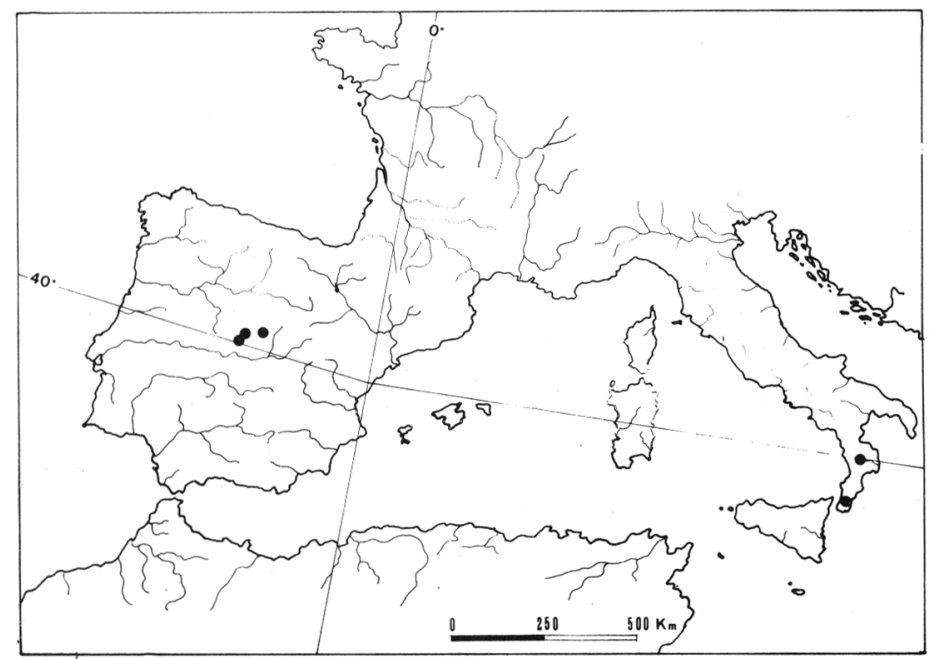

Fig. 3.- Mapa de distribución de los ejemplares analizados de Umbilicaria hirsuta.

El área de distribución de $U$. freyi en la Península Ibérica es similar al de $U$. grisea pero con un rango altitudinal mucho más estrecho. Todas las localidades conocidas hasta el momento se sitúan en una banda comprendida entre 1000 y 1500 $\mathrm{m}$ de altitud. Esto significa que $U$. frey $i$ es en muchas localidades simpátrica tanto con $U$. grisea como con $U$. hirsuta (Fig. 4).

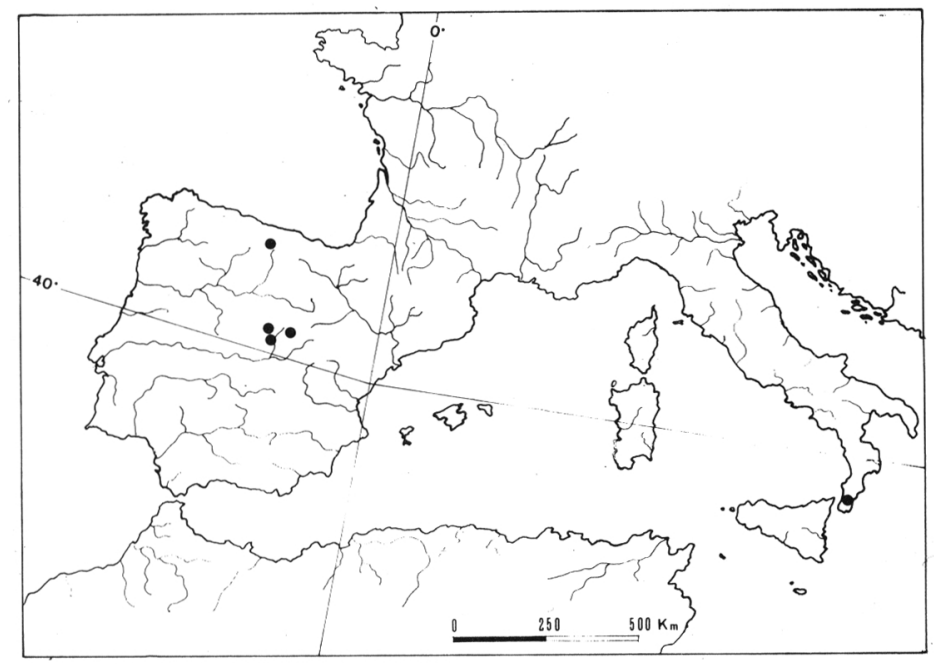

Fig. 4.- Mapa de distribución de los ejemplares analizados de Umbilicaria freyi. 
También en lo que se refiere a la autoecología Umbilicaria grisea y U. hirsuta son las especies más claramente diferenciadas. La primera, de cara inferior glabra (Fig. 1D), vive sobre paredes verticales o extraplomadas, protegidas de la precipitación y nunca afectadas por escorrentías. Es por lo tanto un liquen claramente xerofítico. $U$. hirsuta sin embargo, es una especie sustratohigrófita, con la cara inferior trabeculada (Fig. 1F) y cubierta de rizinomorfos, que tiende a ocupar escorrentías más o menos esporádicas procedentes tanto de la fusión de la nieve como del agua de lluvia. U. freyi tiene un comportamiento intermedio entre las dos especies mencionadas. Su plasticidad morfológica le permite colonizar tanto paredes secas como escorrentías, según se trate de ecotipos glabros o con rizinomorfos, respectivamente (Fig. 1E).

\section{RESULTADOS DE LOS ANÁLISIS QUÍMICOS}

Los análisis mediante HPLC nos han permitido obtener resultados semicuantitativos correlacionables con cada una de las especies estudiadas (Tabla 1). En estos resultados se observa como la capacidad de almacenamiento de ácido umbilicárico es máxima en Umbilicaria freyi y prácticamente nula en $U$. hirsuta. Esta sustancia, exclusiva del género Umbilicaria, se presenta en concentraciones relativas muy constantes en cada una de las especies estudiadas. El ácido girofórico aparece con elevada concentración en todas ellas, apreciándose, en todo caso, una disminución de su porcentaje relativo en $U$. grisea y $U$. freyi, en concordancia con el aumento de ácido umbilicárico. La concentración de ácido lecanórico parece, por el contrario, independiente de la de ácido umbilicárico, por lo que el cociente entre sus porcentajes relativos puede utilizarse como valor discriminante (Tabla 1). Este cociente es siempre menor que 0.5 en $U$. freyi, cercano a 1 en $U$. grisea y superior a 5 en $U$. hirsuta .

\begin{tabular}{lccr}
\hline Sustancia & U. hirsuta & U. grisea & U.freyi \\
\hline ác. lecanórico & $3.32 \pm 0.84$ & $4.27 \pm 1.19$ & $5.55 \pm 0.95$ \\
ác. umbilicárico & $0.42 \pm 0.49$ & $2.76 \pm 2.44$ & $10.32 \pm 3.98$ \\
ác. girofórico & $92.05 \pm 2.46$ & $91.07 \pm 2.85$ & $80.59 \pm 4.80$ \\
ác. lec./ ác. umb. & 7.90 & 1.54 & 0.53 \\
\hline
\end{tabular}

Tabla 1. Porcentaje de los ácidos lecanórico, umbilicárico y girofórico en Umbilicaria hirsuta, $U$. grisea y $U$. freyi.

Además de estos ácidos liquénicos claramente identificados, aparecen en los cromatogramas (Fig. 5. Tablas 2 y 3 ) una serie de picos no identificados. 


\begin{tabular}{cccc}
\hline Sustancia & N & tR & Desviación estándar \\
\hline 1 & 29 & 4.31 & 0.08 \\
2 & 26 & 5.09 & 0.10 \\
3 & 30 & 5.87 & 0.10 \\
4 & 22 & 6.71 & 0.05 \\
5 & 7 & 6.93 & 0.10 \\
6 & 8 & 7.68 & 0.09 \\
7 & 27 & 7.99 & 0.24 \\
8 & 30 & 11.83 & 0.38 \\
\hline
\end{tabular}

Tabla 2. Valores medios del tiempo de retención de cada una de las sustancias. $\mathrm{N}=\mathrm{n}^{\circ}$ de muestras en las que aparece cada una de las sustancias. 3= ácido lecanórico; 7 =ácido umbilicárico; 8= ácido girofórico; $1,2,4,5,6=$ sustancias no identificadas. $\mathrm{tR}=$ tiempo de retención en minutos (valor medio).

\begin{tabular}{ccccccccc}
\hline & \multicolumn{7}{c}{ SUSTANCIAS DETECTADAS } \\
\hline Talos & 1 & 2 & 3 & 4 & 5 & 6 & 7 & 8 \\
\hline 1 & 0.20 & 0.00 & 3.80 & 0.60 & 0.00 & 0.00 & 0.00 & 94.40 \\
2 & 1.70 & 0.30 & 3.60 & 0.20 & 0.00 & 6.50 & 0.00 & 89.30 \\
3 & 2.00 & 0.80 & 4.00 & 0.90 & 0.90 & 0.30 & 0.00 & 90.90 \\
4 & 2.30 & 0.60 & 3.00 & 0.00 & 0.00 & 0.70 & 0.00 & 93.50 \\
5 & 2.00 & 0.20 & 2.00 & 0.00 & 0.30 & 0.00 & 1.00 & 94.50 \\
6 & 2.50 & 0.50 & 2.50 & 0.30 & 0.40 & 0.40 & 1.10 & 92.40 \\
7 & 1.90 & 0.00 & 3.10 & 0.20 & 0.20 & 0.70 & 0.40 & 93.40 \\
8 & 3.70 & 0.90 & 4.60 & 0.50 & 0.50 & 0.90 & 0.90 & 87.80 \\
9 & 2.79 & 0.85 & 6.14 & 0.00 & 0.00 & 0.00 & 6.16 & 84.06 \\
10 & 2.29 & 0.66 & 6.37 & 0.00 & 0.00 & 0.00 & 9.50 & 81.17 \\
11 & 2.39 & 0.63 & 4.33 & 0.00 & 0.00 & 0.00 & 8.57 & 84.03 \\
12 & 2.35 & 0.28 & 5.25 & 0.00 & 0.00 & 0.00 & 13.84 & 77.87 \\
13 & 2.18 & 0.34 & 4.06 & 0.19 & 0.00 & 0.00 & 6.63 & 86.59 \\
14 & 3.21 & 1.08 & 6.05 & 0.24 & 0.00 & 0.00 & 7.98 & 81.43 \\
15 & 3.47 & 0.78 & 6.67 & 0.12 & 0.00 & 0.00 & 17.77 & 71.17 \\
16 & 2.74 & 0.89 & 5.53 & 0.30 & 0.00 & 0.00 & 12.14 & 78.40 \\
\hline 17 & 1.17 & 0.52 & 6.33 & 0.89 & 0.00 & 0.87 & 0.76 & 89.45 \\
18 & 0.41 & 0.38 & 3.74 & 0.51 & 0.00 & 0.00 & 1.60 & 93.28 \\
19 & 0.92 & 0.36 & 3.41 & 0.00 & 0.38 & 0.18 & 0.54 & 94.10 \\
20 & 0.86 & 0.16 & 2.52 & 0.00 & 0.30 & 0.00 & 1.32 & 94.83 \\
21 & 0.48 & 0.33 & 3.70 & 0.39 & 0.00 & 0.00 & 4.12 & 90.97 \\
22 & 0.79 & 0.19 & 4.30 & 0.42 & 0.00 & 0.00 & 7.93 & 86.27 \\
23 & 1.51 & 0.71 & 4.97 & 0.29 & 0.00 & 0.00 & 2.23 & 90.28 \\
24 & 1.46 & 0.00 & 5.23 & 0.23 & 0.00 & 0.00 & 3.65 & 89.43 \\
\hline & & & & & & & &
\end{tabular}

Tabla 3. Porcentaje de las sustancias encontradas en los distintos talos analizados. Sustancias detectadas: $3=$ ácido lecanórico; $7=$ ácido umbilicárico; 8= ácido girofórico; 1, 2, 4, 5, 6= sustancias no identificadas. Talos analizados: 1-8, Umbilicaria hirsuta; 9-16, U. freyi; 17-24, U. grisea. 

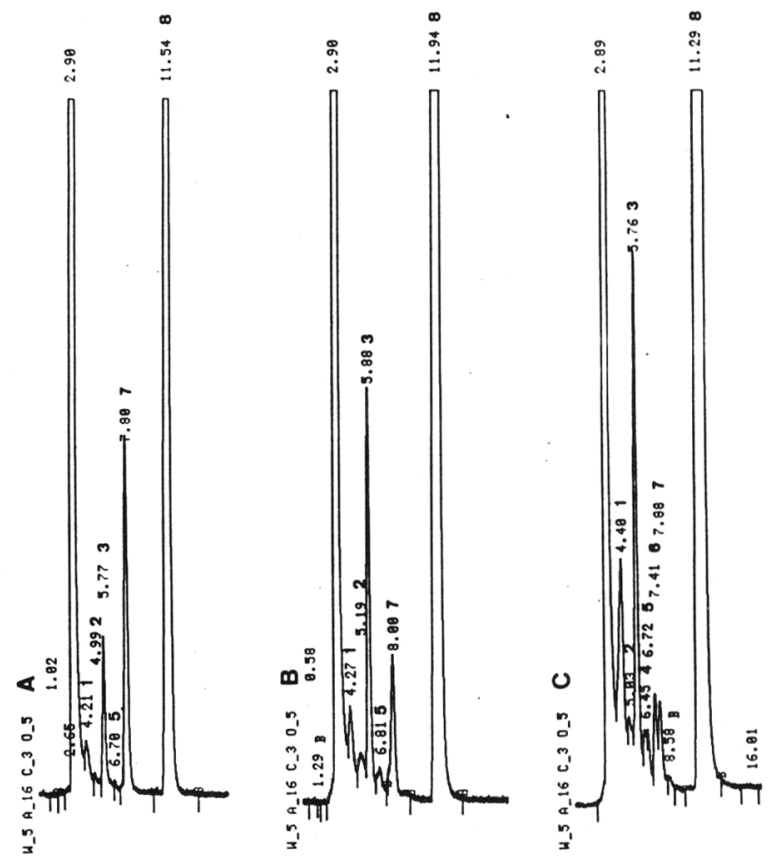

Fig. 5.- Cromatogramas obtenidos por HPLC de Umbilicaria freyi (A), U. grisea (B) y U. hirsuta (C). 3= ácido lecanórico; 7= ácido umbilicárico; 8= ácido girofórico; 1, 2, 4, 5, 6= picos no identificados.

\section{DISCUSIÓN}

Como se desprende de los resultados morfológicos y anatómicos, Umbilicaria freyi es un taxon intermedio entre $U$. hirsuta y $U$. grisea, cuyo único carácter diferencial constante es la presencia de esquizidios en la cara superior del talo. Sin embargo, estos propágulos vegetativos están ontogénicamente muy relacionados con los parasoredios de las dos especies afines (Codogno \& al., 1990), por lo que la diferenciación específica de $U$. freyi es en algunos casos problemática. En este caso los caracteres químicos contribuyen decisivamente a reforzar la caracterización taxonómica de las tres especies, sobre todo en lo que respecta a $U$. freyi, claramente diferenciada por su alto porcentaje relativo de ácido umbilicárico, sustancia mucho menos abundante en $U$. grisea y casi indetectable en $U$. hirsuta (Tabla 1 ). El análisis de componentes principales (Fig. 6) muestra también una fuerte segregación de $U$. freyi con respecto a las otras dos especies. El cociente entre ácido lecanórico y ácido umbilicárico puede utilizarse también como valor discriminante (Tabla 1). Anteriormente $U$. hirsuta y $U$. grisea habían sido analizadas sólo mediante técnicas de TLC, que únicamente habían permitido reconocer la presencia de ácido girofórico (Dahl \& Krog, 1973; Lisická, 1980). 


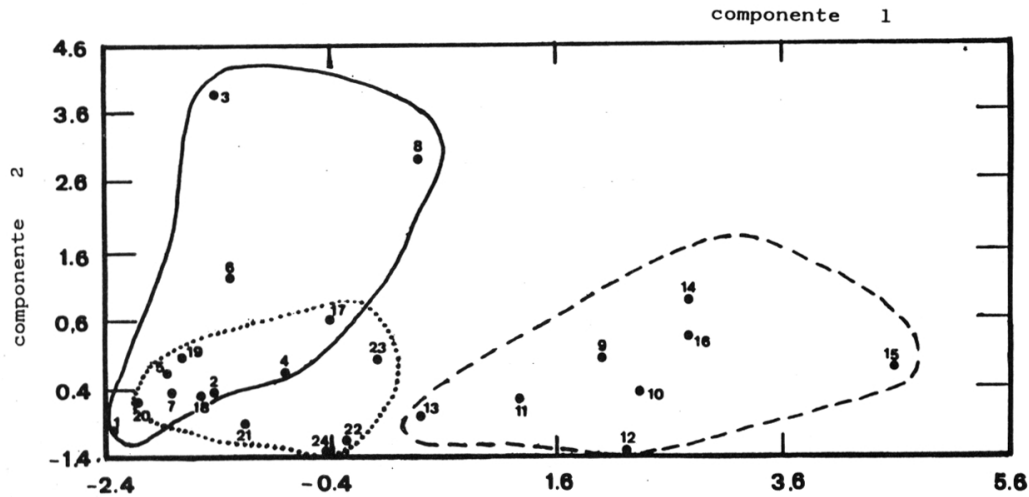

Fig. 6.- Segregación mediante el análisis de componentes principales de los talos analizados de Umbilicaria freyi (- - ), U. grisea ( . . . . ) y U. hirsuta ( _ _ ).

Atendiendo a su estructura química (Fig. 7), el ácido umbilicárico podría derivar directamente por metilación del ácido girofórico (Lawrey, 1977). Esto explicaría la proporción inversa de sus porcentajes relativos en las tres especies analizadas. El ácido lecanórico, posible precursor del ácido girofórico, se mantiene, por el contrario, más o menos constante. La mayor parte de las sustancias desconocidas podrían estar involucradas en la síntesis o degradación de los ácidos lecanórico y umbilicárico.

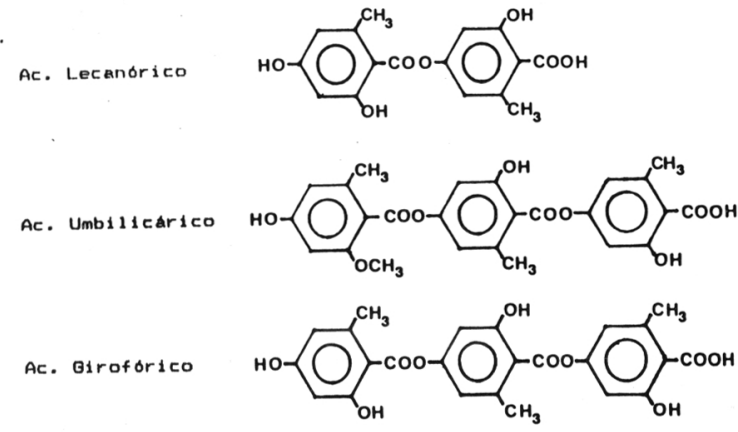

Fig. 7.- Estructura química de las sustancias identificadas.

Los caracteres morfológicos y ecológicos intermedios de Umbilicaria freyi sugieren la hipótesis de que sea éste el taxon más primitivo del grupo, a partir del cual y mediante un proceso de reducción de los esquizidios, habrían evolucionado, tal vez de forma simpátrica, las otras dos especies. También su autoecología, variable en cuanto a la dependencia de agua líquida, y su distribución altitudinal, restringida a una 
estrecha banda supramediterránea (montana) de condiciones climáticas moderadas, podrían considerarse basales con respecto a las más especializadas de $U$. hirsuta y $U$. grisea. Por otra parte, su distribución geográfica sugiere una vez más la importancia del Mediterráneo Occidental como área de especiación para muchos táxones de la familia Umbilicariaceae (Sancho \& Crespo, 1989).

A esta hipótesis filogenética los resultados de los análisis químicos aportarían la evidencia de una gradación en las concentraciones de ácido umbilicárico, pero, por el momento, no es posible saber si la aparición de esta sustancia en mayor o menor proporción debe considerarse un carácter derivado o primitivo. Lawrey (1977) encuentra que aquellos compuestos oximetilados aparecen con mayor frecuencia en géneros más avanzados desde el punto de vista morfológico. No obstante, en el género Umbilicaria, estas consideraciones necesitarían una comprobación mucho más profunda, estudiando comparativamente un mayor número de especies.

\section{AGRADECIMIENTOS}

La financiación para este trabajo ha sido aportada por la Comisión Interministerial de Ciencia y Tecnología Proyecto número PB87-0229.

\section{BIBLIOGRAFÍA}

CODOGNO, M., J. POELT \& D. PUNTILLO -1989- Umbilicaria freyi spec. nova und der Formenkreis von Umbilicaria hirsuta in Europa (Lichenes, Umbilicariaceae). Pl. Syst. Evol. 165: 55-69.

CODOGNO, M. \& L.G. SANCHO -1990- Distribution patterns of the lichen family Umbilicariaceae in the W-Mediterranean basin. Sixth Meting, Delphi 10-16 Sept. 1989. In press.

DAHL, E. \& H. KROG -1973- Macrolichens. Scand. Univ. Books, Oslo, Bergen, Tromso.

EGEA, J.M. \& X. LLIMONA -1981- Liquenes silicícolas de la Sierra de los Filabres y Sierra Alhamilla (Almería, España). Anales de la Universidad de Murcia Ciencias. 37 (1-4): 107 152.

HLADUN, N. -1982-Aportación a la flora, morfología y vegetación de los liquenes silicícolas de la parte alta del Montseny (Cataluña). Universitat de Barcelona.

LAWREY, J.D. -1977- Adaptative significance of O-Methylated lichen Depsides and Depsidones. Lichenologist, 9: 137-142.

POELT, J. -1977- Die Gattung Umbilicaria. Khumbu Himal, Ergebnisse Forschungsunternehmens Nepal Himalaya 6: 397-435.

RIVAS-MARTíNEZ, S. -1988- Memoria del Mapa de Series de Vegetación de España 1: 400.000. Madrid. ICONA.

SANCHO, L.G. \& A. CRESPO -1989-Lasallia hispanica and related species. Lichenologist, $21(1): 45-58$.

WIRTH, V. -1980- Flechtenflora. Eugen Ulmer Verlag. Stuttgart.

(Aceptado para su publicación en Junio de 1.990)

Dirección de los autores: L.G. SANCHO \& E. MANRIQUE: Dpto. de Biología Vegetal II, Facultad de Farmacia, Universidad Complutense, 28040 Madrid. E. SERIÑ Á \& R. ARROYO: Dpto. de Biología Vegetal I, Facultad de Biología, Universidad Complutense, 28040 Madrid. 